03

\title{
Влияние перпендикулярного электростатического поля на вызванный аэродинамическими силами первичный распад тонкой плоской струи жидкого диэлектрика
}

\author{
(C) B.М. Коровин, ${ }^{1}$ B.A. Кажан ${ }^{2, \uparrow}$ \\ ${ }^{1}$ Научно-исследовательский институт механики Московского государственного университета им. М.В. Ломоносова, \\ 119192 Москва, Россия \\ ${ }^{2}$ Российский государственный аграрный университет - МСХА им. К.А. Тимирязева, \\ 127550 Москва, Россия \\ ฯ e-mail: verazhan@yandex.ru
}

(Поступило в Редакцию 9 мая 2018г.)

Изучен распад плоской струи неэлектропроводной жидкости с большой диэлектрической проницаемостью. Струя движется с постоянной скоростью в покоящемся на бесконечности газе при наличии перпендикулярного электростатического поля. В начальный момент границы струи параллельны. Ее толщина много меньше капиллярной постоянной. В числе определяющих параметров учтена плотность газа. Рассмотрен случай, когда аэродинамические силы, возникающие при волновом возмущении поверхностей раздела жидкость-газ вблизи среза сопла, вносят бо́льший вклад в динамику жидкости, чем капиллярные силы. Показано, что при аэродинамической неустойчивости струи дополнительное воздействие перпендикулярного электростатического поля умеренной напряженности вызывает существенное уменьшение характерного размера фрагментов, образовавшихся после распада струи.

DOI: 10.21883/JTF.2019.03.47170.182-18

\section{Введение}

Распыление жидкостей, возникающее под действием аэродинамических сил, широко используется в технических устройствах различного назначения $[1,2]$. В случае плоских струй исследование этого явления на базе уравнений Навье-Стокса проводят путем численного моделирования развития по времени трехмерных возмущений [3].

В отечественных работах при моделировании распыления струй топлива [4] после расчета формы струи последовательного используют ранее созданные модели. Эти модели описывают в линейной постановке: а) первичный распад струи на фрагменты, б) вторичный распад фрагментов на капли, в) дробление капель. Ранее аналогичная схематизация была использована при аналитическом исследовании аэродинамической неустойчивости плоской струи [5].

Впервые экспериментальное исследование механизма распада приближенно осесимметричного тонкого слоя жидкости, движущегося с достаточно большой скоростью в покоящемся вдали от жидкости газе, было выполнено в работе [6]. После выхода жидкости из сопла специальной конструкции жидкая конфигурация имела расширяющуюся в направлении движения коническую полость. Считалось, что при удалении индивидуальных жидких объемов от среза сопла аэродинамические силы вызывают распад слоя на тонкие кольца. Характерный размер капель, образовавшихся на финальной стадии развития неустойчивости движущихся в газе жидких колец, вычислялся с использованием рэлеевской теории неустойчивости жидкого цилиндра [7].
Работа [8] положила начало аналитическим исследованиям первичного распада тонкой плоской струи (толщина струи много меньше капиллярной постоянной) невязкой жидкости. В [8] изучено развитие плоских волн, гребни которых перпендикулярны направлению движения струи. Было предложено разделять такие волны на антисимметричные и симметричные относительно плоскости, делящей пополам постоянную в начальный момент толщину струи. Обоснование такого подхода, широко используемого при исследовании динамики тонких жидких слоев с параллельными границами, приведено в [9] для капиллярных волн. В [8] показано, что наиболее быстро растущая волна антисимметрична. Ее длина определяет характерный размер фрагментов, образовавшихся после первичного распада струи. Исследовано влияние определяющих параметров на длину и скорость роста этой волны.

Экспериментальные данные по развитию неустойчивости и распаду тонкой плоской струи, вызванному аэродинамическими силами, впервые были приведены в [10]. Эти данные сравнены с теоретическими результатами, полученными в [10] из решения задачи, близкой по постановке к задаче, рассмотренной в [8].

В работе [11] в плоской постановке изучен вызванный развитием антисимметричных длинных волн первичный распад тонкой плоской струи невязкой диэлектрической жидкости, движущейся в потоке воздуха при наличии однородного продольного электростатического поля. Различные по величине скорости жидкости и газа параллельны полю. Рассматриваемые среды не содержат свободных электрических зарядов. Наряду с аэродинами- 
ческими и капиллярными силами учтены поверхностные пондеромоторные силы. Показано, что распад струи вызывается аэродинамическими силами и неустойчивостью Кельвина-Гельмгольца. При этом пондеромоторные силы оказывают стабилизирующее воздействие.

Следует отметить, что устойчивое (с учетом сил поверхностного натяжения) совместное течение в поле тяжести жидкого диэлектрика и газа с разрывом скорости на поверхности раздела [12] теряет устойчивость при включении достаточно сильного перпендикулярного электростатического поля [13].

В работе [14] исследовано влияние определяющих параметров на развитие плоских и пространственных симметричных и антисимметричных волн при движении тонкого плоского слоя диэлектрической жидкости в покоящемся вдали от жидкости газе при наличии продольного электростатического поля. В постановке задачи учтено изменение давления в газе вблизи границ слоя. В отличие от [11] рассмотрена эволюция как длинных, так и коротких волн.

В настоящей работе изучен созданный аэродинамическими силами первичный распад тонкой плоской струи жидкого диэлектрика, не содержащего свободных электрических зарядов. Струя движется параллельно обкладкам заряженного воздушного конденсатора. Показано, что при умеренной напряженности электрического поля и большой диэлектрической проницаемости жидкости пондеромоторные силы, локализованные на границах слоя, вызывают существенное уменьшение характерного размера фрагментов, образовавшихся после распада струи.

\section{Уравнения и граничные условия}

Система уравнений и граничных условий электрогидродинамики, описываемая моделью Тейлора-Мелчера $[15,16]$, представляет результаты экспериментальных и теоретических исследований, проведенных во второй половине $\mathrm{XX}$ века. Эта модель учитывает как уход из рассматриваемого объема жидкого диэлектрика свободных электрических зарядов, образующихся в результате различных физикохимических процессов в объеме и на его границе, так и поступление в этот объем свободных зарядов извне.

Наряду с моделью Тейлора-Мелчера в электрогидродинамике используется более простая модель однородного по температуре (и другим физическим характеристикам) несжимаемого жидкого диэлектрика, не содержащего свободных электрических зарядов. Теоретические основы такого подхода были заложены в конце XIX века [17]. В рамках этой модели запись пондеромоторных сил является частным случаем общих формул электродинамики сплошных сред [18].

В однородном несжимаемом жидком диэлектрике при отсутствии свободных электрических зарядов объемные пондеромоторные силы отсутствуют. Ввиду этого в

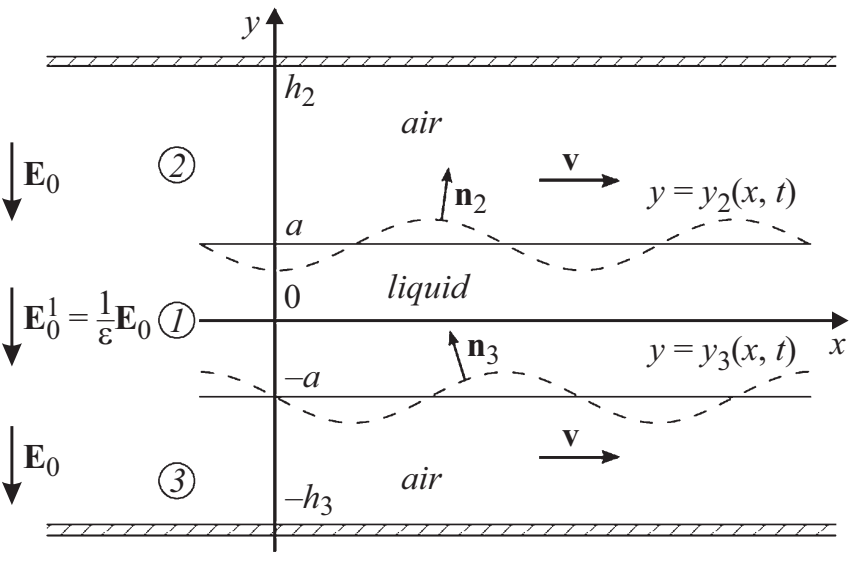

Рис. 1. Геометрия задачи и обозначения.

такой модели электрическое поле оказывает силовое воздействие на жидкость лишь за счет пондеромоторных сил, локализованных на поверхности раздела с газом или с жидкостью, имеющей другую диэлектрическую проницаемость.

В работе рассматривается плоскопараллельное движение струи невязкой несжимаемой диэлектрической жидкости между пластинами заряженного воздушного конденсатора. Струя находится на значительном удалении от пластин. Жидкость не содержит свободных электрических зарядов. Как и в работах $[3,5,8-11,14]$ силой тяжести пренебрегается.

Предполагается, что в начальный момент времени $t=0$ границы струи параллельны пластинам, все жидкие частицы имеют одинаковую скорость относительно пластин, а воздух покоится. Толщина струи $2 a$ мала по сравнению с капиллярной постоянной. Движение рассматриваемых сред при $t>0$ считается потенциальным. Сжимаемостью воздуха пренебрегается.

Как и в задаче об обтекании волнистой стенки однородным потоком газа [19], введем декартову систему координат $x, y$, в которой вдали от струи скорость $\mathbf{v}$ однородного воздушного потока направлена вдоль оси $x$, параллельной пластинам (рис. 1), а жидкие частицы в момент времени $t=0$ покоятся. Обозначим через $\mathbf{a}_{x} \mathbf{a}_{y}$ соответствующие орты.

Наличие возникающих возмущений вызывает изменение границ струи с ростом $t$. Пусть уравнения $y=y_{2}(x, t), y=y_{3}(x, t), t>0$, описывают соответственно форму верхней и нижней поверхностей раздела жидкость-воздух. Будем отмечать индексами $y=1,2,3$ физические величины, относящиеся к жидкости $(j=1)$ и к воздуху, находящемуся сверху $(j=2)$ и снизу $(j=3)$.

Обозначим через $\varphi_{j}(x, y, t), u_{j}(x, y, t)$ потенциалы скоростей $\mathbf{v}_{j}(x, y, t)=\nabla \varphi_{j} \quad$ и электрических полей $\mathbf{E}_{j}(x, y, t)=-\nabla u_{j}$. Эти функции должны удовлетворять 
уравнениям Лапласа

$\nabla^{2} \varphi_{j}=0, \quad \nabla^{2} u_{j}=0, \quad j=1,2,3 \quad \nabla=\frac{\partial}{\partial x} \mathbf{a}_{x}+\frac{\partial}{\partial y} \mathbf{a}_{y}$.

На поверхностях раздела потенциал электрического поля и нормальная составляющая вектора индукции непрерывны

$$
\begin{aligned}
& y=y_{2}(x, t): \quad u_{1}=u_{2}, \quad \varepsilon \frac{\partial u_{1}}{\partial n_{2}}=\frac{\partial u_{2}}{\partial u_{2}}, \\
& y=y_{3}(x, t): \quad u_{1}=u_{3}, \quad \varepsilon \frac{\partial u_{1}}{\partial n_{3}}=\frac{\partial u_{3}}{\partial u_{3}},
\end{aligned}
$$

где $\varepsilon$ - диэлектрическая проницаемость жидкости, а $\mathbf{n}_{l}(x, t)$ - единичные векторы нормалей к поверхностям раздела $y=y_{1}(x, t), l=2,3$.

Потенциал верхней пластины фиксирован, а нижняя пластина заземлена

$$
y=h_{2}: \quad u_{2}=u_{0}, \quad y=-h_{3}: \quad u_{3}=0 .
$$

При параллельных поверхностях раздела $y= \pm a$ :

$$
\varphi_{1}^{0}, \quad \varphi_{2}^{0}(x)=\varphi_{3}^{0}(x)=v x,
$$

а задача электростатики (1)-(4) имеет решение

$$
\begin{gathered}
u_{1}^{0}(y)=E_{1}^{0}\left[y+\varepsilon h_{3}-a(\varepsilon-1)\right], \\
u_{2}^{0}(y)=E_{1}^{0}\left[\varepsilon\left(y+h_{3}\right)-2 a(\varepsilon-1)\right], \\
u_{3}^{0}=E_{0}\left(y+h_{3}\right), \\
E_{0}=\frac{\varepsilon u_{0}}{\varepsilon\left(h_{2}+h_{3}\right)-2 a(\varepsilon-1)}, \quad E_{1}^{0}=\frac{1}{\varepsilon} E_{0} .
\end{gathered}
$$

Поскольку струя тонкая, то $2 a(\varepsilon-1) /\left(\varepsilon h_{3}\right)=1$. Пренебрегая членами указанного и более высокого порядков малости, получаем

$$
\begin{gathered}
E_{0}=\frac{u_{0}}{h_{2}+h_{3}}, \quad u_{1}^{0}(y)=\left(y+\varepsilon h_{3}\right) E_{1}^{0}, \\
u_{2}^{0}(y)=u_{3}^{0}(y)=E_{0}\left(y+h_{3}\right) .
\end{gathered}
$$

Поле $E_{0}$, естественно, не может превышать пробивное значение $E_{b}$. Для сухого воздуха $E_{b}=30 \mathrm{kV} / \mathrm{cm}$ [17]. В настоящей работе, как и в [17], в качестве основной системы единиц выбрана система CGS.

Для вычисления давлений $p_{j}(x, y, t)$ используются интегралы Коши-Лагранжа

$$
p_{j}=-\rho_{j}\left[\frac{\partial \varphi_{j}}{\partial t}+\frac{1}{2}\left(\nabla \varphi_{j}\right)^{2}\right]+C_{j}, \quad j=1,2,3,
$$

где $\rho_{1}-$ плотность жидкости, $\rho_{2}=\rho_{3}-$ плотность воздуха, $C_{j}$ - константы.

Вдали от струи воздушный поток однороден

$$
y / a \rightarrow+\infty: \quad \nabla \varphi_{2} \rightarrow \mathbf{v}, \quad y / a \rightarrow-\infty: \quad \nabla \varphi_{3} \rightarrow \mathbf{v} .
$$

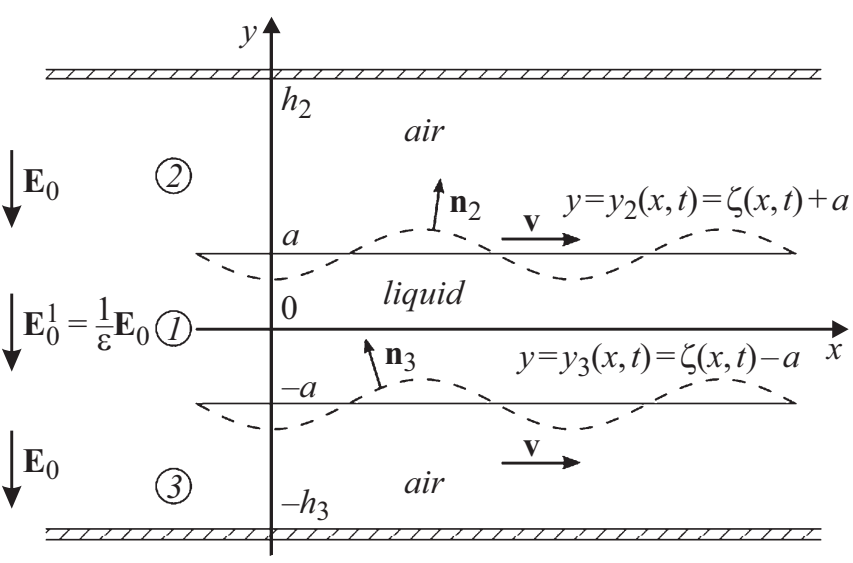

Рис. 2. Антисимметричные волны.

На поверхностях раздела должны выполняться кинематические и динамические условия

$$
\begin{gathered}
y=y_{2}(x, t): \quad \frac{\partial y_{2}}{\partial t}+\frac{\partial \varphi_{1}}{\partial x} \frac{\partial y_{2}}{\partial x}=\frac{\partial \varphi_{1}}{\partial y}, \\
\frac{\partial y_{2}}{\partial t}+\frac{\partial \varphi_{2}}{\partial x} \frac{\partial y_{2}}{\partial x}=\frac{\partial \varphi_{2}}{\partial y}, \\
y=y_{2}(x, t): \\
p_{1}-p_{2}=\frac{\alpha}{R_{2}}-\frac{\varepsilon-1}{8 \pi}\left[\left(\nabla u_{1}\right)^{2}+(\varepsilon-1)\left(\frac{\partial u_{1}}{\partial n_{2}}\right)^{2}\right], \\
y=y_{3}(x, t): \quad \frac{\partial y_{3}}{\partial t}+\frac{\partial \varphi_{1}}{\partial x} \frac{\partial y_{3}}{\partial x}=\frac{\partial \varphi_{1}}{\partial y}, \\
\frac{\partial y_{3}}{\partial t}+\frac{\partial \varphi_{3}}{\partial x} \frac{\partial y_{3}}{\partial x}=\frac{\partial \varphi_{3}}{\partial y}, \\
y=y_{3}(x, t): \\
p_{1}-p_{3}=\frac{\alpha}{R_{3}}-\frac{\varepsilon-1}{8 \pi}\left[\left(\nabla u_{1}\right)^{2}+(\varepsilon-1)\left(\frac{\partial u_{1}}{\partial n_{3}}\right)^{2}\right],
\end{gathered}
$$

где $\alpha-$ коэффициент поверхностного натяжения, а $r_{l}-$ радиусы кривизны плоских кривых $y_{l}=y_{l}(x, t), l=2,3$, зависящих от параметра $t$. Пропорциональные $\varepsilon-1$ слагаемые в правых частях динамических условий (7), (9) описывают вклад поверхностных пондеромоторных сил [18].

После линеаризации электрогидродинамической задачи (1)-(9) исследуется воздействие пондеромоторных сил на первичный распад струи, реализующийся для рассматриваемой жидкости при $E_{0}=0$.

Используется следующая схематизация развития волновых возмущений, различающихся между собой длинами волн. Предполагается, что на каждой из первоначально плоских поверхностей раздела $y= \pm a$ возникает только одна гармоническая волна. Пусть эта волна описывается функцией $\xi(x, t)=Z \cos (k x-\omega t)$. Аналогичное представление вертикального смещения поверхности раздела использовано в [12] при исследовании 
устойчивости тангенциального разрыва в поле тяжести с учетом поверхностного натяжения.

В обозначениях, введенных выше, $y_{2}(x, t)=a+$ $+\zeta(x, t) . \quad$ В случае $y_{2}(x, t)=-y_{2}(x, t)$ волну называют симметричной, а в случае $y_{3}(x, t)=-a+$ $+\zeta(x, t)$ антисимметричной (рис. 2$)$.

В рамках линейной задачи взаимодействие волн не учитывается. Ввиду этого допускается принятая схематизация.

\section{Дисперсионное уравнение в случае антисимметричных волн}

Положим

$$
\begin{array}{r}
\varphi_{l}(x, y, t)=v x+f_{l}(x, y, t), \quad l=2,3, \\
u_{j}(x, y, t)=u_{j}^{0}(y)+\psi_{j}(x, y, t), \quad j=1,2,3 .
\end{array}
$$

Гармонические функции $\varphi_{1}(x, y, t), f_{1}(x, y, t)$ являются потенциалами малых возмущений скорости. Производные $\frac{\partial \psi_{j}}{\partial x}, \frac{\partial \psi_{j}}{\partial y}$ выражают компоненты векторов возмущений электрических полей. Предполагается, что эти величины малы по сравнению с напряженностями полей в соответствующих областях при плоских границах слоя.

Линеаризованные интегралы Коши-Лагранжа (5) и кинематические условия (6), (8) записываются следующим образом:

$$
\begin{gathered}
p_{1}=-\rho_{1} \frac{\partial \varphi_{1}}{\partial t}+C_{1}, \\
p_{l}=-\rho_{l}\left(\frac{v^{2}}{2}+\frac{\partial f_{1}}{\partial t}+v \frac{\partial f_{l}}{\partial x}\right)+C_{l}, \quad l=2,3, \\
y=a: \quad \frac{\partial \xi}{\partial t}=\frac{\partial \varphi_{1}}{\partial y}, \quad \frac{\partial \xi}{\partial t}+v \frac{\partial \xi}{\partial x}=\frac{\partial f_{2}}{\partial y}, \\
y=-a: \quad \frac{\partial \xi}{\partial t}=\frac{\partial \varphi_{1}}{\partial y}, \quad \frac{\partial \xi}{\partial t}+v \frac{\partial \xi}{\partial x}=\frac{\partial f_{3}}{\partial y},
\end{gathered}
$$

Предполагается, что $\nabla f_{2} \rightarrow 0$ при $y / a \rightarrow+\infty$ и $\nabla f_{3} \rightarrow 0$ при $y / a \rightarrow-\infty$.

После линеаризации граничных условий задачи электростатики (1)-(4) получаем

$$
\begin{gathered}
\frac{\partial^{2} \psi_{j}}{\partial x^{2}}+\frac{\partial^{2} \psi_{j}}{\partial y^{2}}=0, \quad j=1,2,3, \\
y=a: \quad \psi_{1}-\psi_{2}=(\varepsilon-1) E_{1}^{0} \xi, \quad \varepsilon \frac{\partial \psi_{1}}{\partial y}=\frac{\partial \psi_{2}}{\partial y}, \\
y=-a: \quad \psi_{1}-\psi_{3}=(\varepsilon-1) E_{1}^{0} \xi, \quad \varepsilon \frac{\partial \psi_{1}}{\partial y}=\frac{\partial \psi_{3}}{\partial y}, \\
y / a \rightarrow+\infty: \quad \psi_{2} \rightarrow 0, \quad y / a \rightarrow-\infty: \quad \psi_{3} \rightarrow 0 .
\end{gathered}
$$

Линеаризуем динамические условия (7), (9). С учетом (10), (11) имеем

$$
\begin{gathered}
y=a: \\
\rho_{2}\left(\frac{\partial f_{2}}{\partial x}+v \frac{\partial f_{2}}{\partial x}\right)-\rho_{1} \frac{\partial \varphi_{1}}{\partial t}=-\alpha \frac{\partial^{2} \xi}{\partial x^{2}}-\frac{\varepsilon-1}{4 \pi} E_{0} \frac{\partial \psi_{1}}{\partial y}
\end{gathered}
$$

$$
\begin{gathered}
y=-a: \\
\rho_{2}\left(\frac{\partial f_{3}}{\partial x}+v \frac{\partial f_{3}}{\partial x}\right)-\rho_{1} \frac{\partial \varphi_{1}}{\partial t}=-\alpha \frac{\partial^{2} \xi}{\partial x^{2}}-\frac{\varepsilon-1}{4 \pi} E_{0} \frac{\partial \psi_{1}}{\partial y}
\end{gathered}
$$

Решения задач гидродинамики (1), (12), (13) и электростатики (14) ищем в виде

$$
\begin{gathered}
\varphi_{1}(x, y, t)=\Phi_{1}(y) \sin (k x-\omega t), \\
f_{l}(x, y, t)=\Phi_{l}(y) \sin (k x-\omega t), \quad l=2,3, \\
\Psi_{j}(x, y, t)=\Psi_{j}(y) \cos (k x-\omega t), \quad j=1,2,3 .
\end{gathered}
$$

Нетрудно получить

$$
\begin{gathered}
\Phi_{1}=\frac{\omega \operatorname{sh} k y}{k \operatorname{ch} k a} Z, \quad \Phi_{2}=\frac{f k-\omega}{k} Z \gamma \exp (-k y), \\
\Phi_{3}=\frac{v k-\omega}{k} Z \gamma \exp (k y), \quad \gamma=\exp (k a),
\end{gathered}
$$

$\Psi_{1}=2(\varepsilon-1) E_{1}^{0} Z K \operatorname{ch} k y, \quad \Psi_{2}=(\varepsilon-1) E_{0} Z S \exp (-k y)$,

$$
\Psi_{3}=(\varepsilon-1) E_{0} Z S \exp (k y),
$$

где

$$
\begin{gathered}
K=\frac{q}{m}, \quad S=\frac{s}{m}, \quad q=\gamma\left[\gamma^{2}(\varepsilon+1)+\varepsilon-1\right], \\
s=2 \gamma^{3}-\gamma^{5}(\varepsilon+1)+\gamma(\varepsilon-1), \\
m=\gamma^{4}(\varepsilon+1)^{2}-(\varepsilon-1)^{2} .
\end{gathered}
$$

После подстановки найденных решений в динамическое условие (15) приходим к дисперсионному уравнению

$$
\begin{aligned}
& \left(\rho_{1} \text { th } k a+\rho_{2}\right) \omega^{2}-2 \rho_{2} v k \omega \\
& \left.+k^{2}\left[\rho_{2} v^{2}-\alpha k+\frac{\varepsilon(\varepsilon-1)^{2}}{2 \pi}\left(E_{1}\right)^{0}\right)^{2} K \operatorname{sh} k a\right]=0 .
\end{aligned}
$$

Подстановка полученных выше решений в (16) приводит к такому же результату.

В случае комплексной частоты $\omega_{1,2}(k) \omega_{R}(k) \pm i \omega_{1}(k)$, где $i$ - мнимая единица, имеем

$$
\begin{aligned}
& \cos \left(k x-\omega_{1,2} t\right)=\cos \left(k x-\omega_{R} t\right) \operatorname{ch}\left(\omega_{1} t\right) \\
& \pm i \sin \left(k x-\omega_{R} t\right) \operatorname{sh}\left(\omega_{1} t\right)
\end{aligned}
$$

т. е. при $\omega_{1}(k) \neq 0$ амплитуда волны с волновым числом увеличивается с ростом времени.

Из (17) видно, что электростатическое поле усиливает вызываемое воздушным потоком дестабилизирующее воздействие. Капиллярные силы препятствуют распаду. 


\section{Первичный распад струи жидкости, имеющей большую диэлектрическую проницаемость}

В случае $\varepsilon=1$ дисперсионное уравнение (17) принимает вид

$$
\begin{aligned}
& \left(\rho_{1} \text { th } k a+\rho_{2}\right) \omega^{2}-2 \rho_{2} v k \omega \\
& +k^{2}\left(\rho_{2} v^{2}-\alpha k+\frac{E_{0}^{2}}{4 \pi}\right)=0 .
\end{aligned}
$$

Введем безразмерные переменные $\kappa$ и $\Omega$ и безразмерные параметры: отношение плотностей $r$, число Вебера We и параметр электрогидродинамического взаимодействия $Q$ :

$$
\begin{gathered}
\kappa=k a, \quad \Omega=\frac{a \omega}{v}, \quad r=\frac{\rho_{2}}{\rho_{1}} \ll 1, \\
\mathrm{We}=\frac{\alpha}{\rho_{1} v^{2} a}, \quad Q=\frac{E_{0}^{2}}{4 \pi \rho_{1} v^{2}} .
\end{gathered}
$$

Запишем дисперсионное уравнение (19) в безразмерном виде

$$
(1+r \operatorname{cth} \kappa) \Omega^{2}-2 r \kappa \operatorname{cth} \kappa \Omega+\kappa^{2} \operatorname{cth} \kappa(r-\mathrm{We} \kappa+Q)=0 .
$$

Найдем корни

$$
\begin{array}{r}
\Omega_{1,2}(\kappa)=\frac{1}{1+r \operatorname{cth} \kappa}(r \kappa \operatorname{cth} \kappa \pm \sqrt{D(\kappa)}), \\
D(\kappa)=\kappa^{2} \operatorname{cth} \kappa[-r+(\operatorname{We} \kappa-Q)(1+\operatorname{cth} \kappa)] .
\end{array}
$$

Из (18), (20) следует, что при $D(\kappa)<0$ амплитуда волны с безразмерным волновым числом увеличивается с ростом времени.

Известно, что при $\mathrm{We}=1, Q=0$ вследствие роста амплитуд волн с волновыми числами $r=\kappa=1$ происходит распад струи [8].

Исследуем поведение волн с волновыми числами $r=\kappa=1$ в случае $Q \neq 0$.

Разложив cth $\kappa$ по степеням $\kappa$, в первом приближении получаем

$$
D(\kappa)=\mathrm{We} \kappa^{2}-[r(1-\mathrm{We})+Q] \kappa-r Q .
$$

В интервале $0<\kappa<\kappa_{1}$, где

$$
\begin{aligned}
\kappa_{1} & =\frac{1}{2 \mathrm{We}}\{r(1-\mathrm{We})+Q \\
& \left.+\left[r^{2}(1-\mathrm{We})^{2}+2 r Q(1+\mathrm{We})+Q^{2}\right]^{1 / 2}\right\},
\end{aligned}
$$

имеем $D(\kappa)<0$. При этом $\Omega_{1,2}(\kappa)=r \pm i \sqrt{D(\kappa)}$.

В точке $\kappa_{m}=[r(1-\mathrm{We})+Q] /(2 \mathrm{We}) \quad$ квадратный трехчлен $Z=-D(\kappa)$ принимает наибольшее значение

$$
Z\left(\kappa_{m}\right)=\frac{1}{4 \mathrm{We}}\left[r^{2}(1-\mathrm{We})^{2}+2 r Q(1+\mathrm{We})+Q^{2}\right] .
$$

Таким образом, безразмерная скорость роста наиболее быстро растущей волны выражается формулой $\operatorname{Im} \Omega_{1,2}\left(\kappa_{m}\right)=\sqrt{Z\left(\kappa_{m}\right)}$.

В размерном виде имеем

$$
\omega_{I}\left(k_{m}\right)=\frac{v}{2 a \sqrt{\mathrm{We}}}\left[r^{2}(1-\mathrm{We})^{2}+2 r Q(1+\mathrm{We})+Q^{2}\right]^{1 / 2} .
$$

При этом формула для вычисления длины $\lambda_{m}$ наиболее быстро растущей волны имеет вид

$$
\lambda_{m}=\frac{2 \pi}{k_{m}}=\frac{2 \pi a}{\kappa_{m}}=\frac{4 \pi a \mathrm{We}}{r(1-\mathrm{We})+Q} .
$$

Рассмотрим распад струи этилового спирта толщиной $2 a=2 \cdot 10^{-3} \mathrm{~cm}$. Укажем $[20,21]$ физические свойства этой жидкости: $\varepsilon=26.4, \rho_{1}=0.79 \mathrm{~g} / \mathrm{cm}^{3}, \alpha=22 \mathrm{dyn} / \mathrm{cm}$ на границе с воздухом. Капиллярная постоянная равна $0.24 \mathrm{~cm}$. У воздуха $\rho_{2}=1.225 \cdot 10^{-3} \mathrm{~g} / \mathrm{cm}^{3}$. Полагаем $v=6.5 \cdot 10^{2} \mathrm{~cm} / \mathrm{s}, E_{0}=24 \mathrm{kV} / \mathrm{cm}$.

В рассматриваемом случае

$$
r=1.55 \cdot 10^{-3} . \quad \mathrm{We}=6.59 \cdot 10^{-2}, \quad Q=1.53 \cdot 10^{-3} .
$$

Учитывая (22), имеем:

a) $\kappa_{m}=1.11 \cdot 10^{-2}, \lambda_{m}=0.57 \mathrm{~cm}, \omega_{1}\left(k_{m}\right)=1.83 \cdot 10^{3} \mathrm{~s}^{-1}$ при $E_{0}=0$,

b) $\kappa_{m}=2.26 \cdot 10^{-2}, \lambda_{m}=0.28 \mathrm{~cm}, \omega_{I}\left(k_{m}\right)=3.9 \cdot 10^{3} \mathrm{~s}^{-1}$ при $E_{0}=24 \mathrm{kV} / \mathrm{cm}$.

Таким образом, электростатическое поле вызывает существенное уменьшение длины $\lambda_{m}$ наиболее быстро растущей волны и увеличение скорости ее роста. Величина $\lambda_{m}$ представляет характерный размер фрагментов.

В теории Рэлея капиллярной неустойчивости струи жидкости полагается, что объемлющая струю среда имеет нулевую плотность [7]. При $\rho_{2}=0$ дисперсионное уравнение (19) принимает вид

$$
\rho_{1} \text { th } k a \omega^{2}+k^{2}\left(\frac{E_{0}^{2}}{4 \pi}-\alpha k\right)=0 .
$$

Отсюда находим

$$
\omega_{1,2}(k)= \pm k\left[\frac{c t h k a}{\rho_{1}}\left(\alpha k-\frac{E_{0}^{2}}{4 \pi}\right)^{1 / 2}\right] .
$$

При $E_{0}=0$ струя устойчива, что согласуется с приведенным в [7] результатом. В случае $E_{0} \neq 0$ амплитуды волн при $k<E_{0}^{2} /(4 \pi \alpha)$ возрастают с ростом времени, т.е. длинноволновая электрогидродинамическая неустойчивость имеет беспороговый характер. При $k \geq E_{0}^{2} /(4 \pi \alpha)$ амплитуды волн не изменяются с ростом времени.

\section{Заключение}

В рамках модели однородного по температуре и другим физическим характеристикам несжимаемого жидкого диэлектрика сформулирована система уравнений и 
граничных условий, описывающая в плоской постановке движение струи невязкой диэлектрической жидкости между пластинами заряженного воздушного конденсатора. Толщина струи много меньше капиллярной постоянной. В жидкости и в газе отсутствуют свободные электрические заряды. Силой тяжести пренебрегается. В начальный момент границы струи параллельны пластинам.

Учитывается возникновение аэродинамических сил при появлении волн на поверхностях раздела жидкость-газ. При достаточно большой скорости струи аэродинамические силы вызывают первичный распад. Явление распада характеризуется тремя безразмерными параметрами: отношением плотности газа к плотности жидкости, числом Вебера и параметром электрогидродинамического взаимодействия.

Выведено дисперсионное уравнение для антисимметричных волн, развитие которых в отсутствие электростатического поля приводит к распаду струи.

Детально исследован распад струи этилового спирта. Показано, что воздействие электростатического поля умеренной напряженности вызывает существенное уменьшение длины $\lambda_{m}$ наиболее быстро растущей волны. При этом увеличивается скорость роста этой волны. По порядку величины $\lambda_{m}$ представляет характерный размер фрагментов при первичном распаде струи.

Приведен также результат исследования неустойчивости плоской диэлектрической струи в перпендикулярном электростатическом поле, полученный с использованием рэлеевской постановки задачи о неустойчивости [7].

Работа выполнена при поддержке Российского фонда фундаментальных исследований (проект № 17-01-00037).

\section{Список литературы}

[1] Yang H.Q. // Phys. Fluid. A. 1992. Vol. 4. P. 681-689.

[2] Sirignano W.A. // J. Fluid. Eng. 1993. Vol. 115. P. 345-378.

[3] Zandian A., Sirignano W.A., Hussain F. // Phys. Fluid. 2017. Vol. 29. N 6. P. 062109-1-062109-19

[4] Погребная Т.В., Свириденков А.А., Третьяков В.В. // Модели и методы аэродинамики. Материалы XV Междунар. школы-семинара. МЦНМО. М. 2015. С. 121-122.

[5] Dombrowski N., Johns W.R. // Chem. Eng. Sci. 1963. Vol. 18. P. 203-214.

[6] Jork J.L., Stubbs H.E., Tek M.R. // Trans. ASME. 1953. Vol. 75. N 7. P. $1279-1286$.

[7] Дразин Ф. Введение в теорию гидродинамической устойчивости. М.: Физматлит, 2005. 287 с.

[8] Squire H.B. // Brit. J. Appl. Mech. 1953. Vol. 4. P. 167-169.

[9] Taylor G.I. // Proc. Roy. Soc. Ser. A. 1959. Vol. 253. P. 296-312.

[10] Hagerty W.W., Shea J.F. // J. Appl. Mech. 1955. Vol. 22. N 4. P. 509-514.

[11] El-Sayed M.F. // Phys. Rev. E. 1999. Vol. 60. N 6. P. $7588-7591$.

[12] Ландау Л.Д., Лифиии, Е.М. Гидродинамика. М.: Наука, 1988. $733 \mathrm{c}$.
[13] Коровин В.М. // ЖТФ. 2017. Т. 87. Вып. 9. С. 1306-1311. DOI:10.21883/JTF.2017.09.44902.1674

[14] El-Sayed M.F. // European Phys. J. E. 2004. Vol. 15. P. 443-455. DOI: 10.1140/epje/i 2004-10076-2

[15] Melcher J.R., Taylor G.I. // Annu. Rev. Fluid. Mech. 1969. Vol. 1. P. 111-146.

[16] Saville D.A. // Annu. Rev. Fluid. Mech. 1997. Vol. 29. P. 27-64.

[17] Сивухин Д.В. Электричество. М.: Наука, 1983. 688 с.

[18] Ландау Л.Д., Лифшиц, Е.М. Электродинамика сплошных сред. М.: Наука, 1992. 664 с

[19] Черный Г.Г. Газовая динамика. М.: Наука, 1988. 424 с.

[20] Таблицы физических величин. / Под ред И.К. Кикоина М.: Атомиздат, 1976. 1008 с.

[21] Бэтчелор Джс. Введение в динамику жидкости. М.: Мир, 1973. $758 \mathrm{c}$. 